\title{
Efeito da adubação e do calcário na produção de matéria seca e de óleo essencial de pimenta-longa(1)
}

\author{
Mariangela de Moraes Messias Sousa ${ }^{(2)}$, Francisco José da Silva Lédo ${ }^{(3)}$ e Flávio Araújo Pimentel(3)
}

Resumo - O objetivo deste trabalho foi estudar o efeito de diferentes doses de N, P e K, em solo que recebeu ou não calcário, sobre a produção de matéria seca e de óleo essencial de pimenta-longa (Piper hispidinervum C. DC.). Utilizou-se o delineamento experimental de blocos casualizados, com três repetições. Os tratamentos foram arranjados no esquema fatorial $3 \times 3 \times 3$, com três doses de $\mathrm{N}(0,8 \mathrm{e}$ $16 \mathrm{~g} /$ planta de $\mathrm{N}), \mathrm{P}\left(0,5\right.$ e $10 \mathrm{~g} /$ planta de $\left.\mathrm{P}_{2} \mathrm{O}_{5}\right)$ e $\mathrm{K}\left(0,5\right.$ e $10 \mathrm{~g} /$ planta de $\left.\mathrm{K}_{2} \mathrm{O}\right)$. Aos 6 e 12 meses após o transplantio, foi avaliada a produção de matéria seca de ramos e folhas, e de óleo essencial. As doses de $\mathrm{P}$ promoveram efeito quadrático sobre a produção de matéria seca, com respostas máximas obtidas com 7,1 e 9,6 g/planta de $\mathrm{P}_{2} \mathrm{O}_{5}$, no solo que recebeu ou não calcário, respectivamente. Em solo não calcareado, as doses de $\mathrm{N}$ promoveram efeito quadrático sobre as produções de matéria seca e de óleo essencial, com os pontos de máxima de 10,2 e 9,4 g/planta de N, respectivamente. O P foi o nutriente que promoveu efeito mais pronunciado no aumento da produção de matéria seca. As médias de produção de matéria seca e de óleo essencial foram maiores no ensaio onde o solo foi calcareado (6.451 kg/ha e $167 \mathrm{~L} / \mathrm{ha}$, respectivamente), do que as obtidas no solo não calcareado $(5.003 \mathrm{~kg} / \mathrm{ha} \mathrm{e}$ $145 \mathrm{~L} / \mathrm{ha}$, respectivamente).

Termos para indexação: Piper hispidinervum, absorção de nutrientes, nitrogênio, fósforo, piperácea

\section{Effect of fertilization and limestone application on production of dry matter and essential oil of long pepper}

\begin{abstract}
The aim of this paper was to evaluate the effect of different doses of N, P and K, in a soil with and without limestone, on dry matter and essential oil production of long pepper (Piper hispidinervum C. DC.). The randomized blocks design was used, with three replications, where the treatments were set up on a $3 \times 3 \times 3$ factorial arrangement, with three doses of $\mathrm{N}(0,8$ and $16 \mathrm{~g} /$ plant of $\mathrm{N}), \mathrm{P}\left(0,5\right.$ and $10 \mathrm{~g} /$ plant of $\left.\mathrm{P}_{2} \mathrm{O}_{5}\right)$ and $\mathrm{K}\left(0,5\right.$ and $10 \mathrm{~g} /$ plant of $\left.\mathrm{K}_{2} \mathrm{O}\right)$. At six and twelve months after transplantation, dry matter of branches plus leaves and essential oil total production were evaluated. The $\mathrm{P}$ doses improved a quadratic effect on the production of dry matter, with maximum response obtained with 7.1 and $9.6 \mathrm{~g} /$ plant of $\mathrm{P}_{2} \mathrm{O}_{5}$, on limestoned and non-limestoned soil, respectively. At the non-limestoned soil, the $\mathrm{N}$ doses had a quadratic effect on dry matter and essential oil yield, with maximum points of 10.2 and $9.4 \mathrm{~g} /$ plant of $\mathrm{N}$, respectively. P was the nutrient that had the most emphasized effect upon the increase of dry matter. Average production of dry matter and essential oil were higher in limestoned soil $(6,451 \mathrm{~kg} / \mathrm{ha}$ e $167 \mathrm{~L} / \mathrm{ha}$, respectively) than in non-limestoned soil $(5,003 \mathrm{~kg} / \mathrm{ha}$ and $145 \mathrm{~L} / \mathrm{ha})$
\end{abstract}

Index terms: Piper hispidinervum, nutrient uptake, nitrogen, phosphorus, piperaceae.

(1) Aceito para publicação em 12 de junho de 2000. Parcialmente financiado pelo Department for International Development, Conselho Britânico.

(2) Embrapa-Centro de Pesquisa Agroflorestal do Acre (CPAFAcre), Caixa Postal 392, CEP 69908-970 Rio Branco, AC. Bolsista do CNPq. E-mail: jmsousa@mdnet.com.br

${ }^{(3)}$ Embrapa-CPAF-Acre. E-mail: fledo@cpafac.embrapa.br, flavio@cpafac.embrapa.br

\section{Introdução}

O safrol é um componente químico aromático, empregado na indústria química como fixador de aromas e como agente sinergístico de inseticidas, que possui grande demanda no mercado mundial. Na Amazônia, foi identificada a espécie Piper 
hispidinervum C. DC., vulgarmente chamada de pimenta-longa, como uma planta grande produtora de óleo essencial rico em safrol. A partir de pesquisas realizadas pela Embrapa-Centro de Pesquisa Agroflorestal do Acre (CPAF-Acre) e pelo Museu Paraense Emílio Goeldi, a pimenta-longa vem se tornando importante alternativa econômica para os agricultores tradicionais e famílias extrativistas da Amazônia, especialmente no Estado do Acre (Maia et al., 1987; Silva, 1993; Pimentel et al., 1998; Rocha Neto et al., 1999).

A região Amazônica é caracterizada por apresentar solos profundos, pobres e ácidos, com fortes limitações quanto à fertilidade natural, e onde o $\mathrm{P}$ é $\mathrm{o}$ elemento mais limitante, seguido do K e N. No Acre, tem-se verificado que a acidez nos solos prejudica 0 desenvolvimento da maioria das culturas (Amaral \& Souza, 1998), e pode ocasionar deficiência e toxicidade nutricional. O uso do calcário é um importante insumo na correção da acidez, pois promove aumento no teor de $\mathrm{Ca}$ e $\mathrm{Mg}$, elevação do pH, e aumento da disponibilidade de $\mathrm{P}$ e Mo, além de favorecer a fixação biológica do N (Fageria et al., 1999).

A pimenta-longa é uma planta ainda em fase de domesticação; na literatura disponível há poucas informações sobre adubação mineral no cultivo dessa planta. Avaliações preliminares realizadas por Brasil \& Viégas (1998) sobre a adubação com NPK em pimenta-longa, conduzida no Pará, mostraram que houve aumento da produtividade de matéria seca da parte aérea. Em Piper nigrum L. (pimenta-do-reino), tem-se verificado efeito favorável da adubação NPK na produtividade, em ensaios realizados na região Amazônica (Veloso et al., 1988; Costa et al., 1989).

O objetivo deste trabalho foi avaliar a adubação com diferentes doses de N, P e K, em solo que recebeu ou não calcário, sobre a produtividade de matéria seca de ramos e folhas, e de óleo essencial de pimenta-longa

\section{Material e Métodos}

Os experimentos foram instalados em julho de 1996 , no campo experimental da Embrapa-CPAF-Acre, em Rio Branco, em solo do tipo Argissolo Vermelho-Escuro, textura argilosa, cuja análise química $(0-20 \mathrm{~cm})$ revelou: $\mathrm{pH} 4,85 ; \mathrm{P}, 1,0 \mathrm{mg} / \mathrm{dm}^{3} ; \mathrm{K}, 39,5 \mathrm{mg} / \mathrm{dm}^{3} ; \mathrm{Ca}$, $1,165 \mathrm{cmol}_{\mathrm{c}} / \mathrm{dm}^{3} ; \quad \mathrm{Mg}, 1,185 \mathrm{cmol}_{\mathrm{c}} / \mathrm{dm}^{3} ; \quad \mathrm{Al}$ $1,265 \mathrm{cmol}_{\mathrm{c}} / \mathrm{dm}^{3} ; \mathrm{H}+\mathrm{Al}, 3,715 \mathrm{cmol}_{\mathrm{c}} / \mathrm{dm}^{3}$ e M.O., $12,6 \mathrm{~g} / \mathrm{dm}^{3}$. As médias anuais de temperatura, precipitação pluvial e umidade relativa variaram em torno de $25^{\circ} \mathrm{C}$, $1.700 \mathrm{~mm}$ e $82 \%$, respectivamente. A distribuição de chu- vas é concentrada no período de outubro a março, correspondente a $75 \%$ da precipitação (Relatório..., 1992).

Foram realizados dois experimentos: um, em solo que recebeu calcário, e outro, que não recebeu calcário. No solo calcareado, aplicou-se 1,78 t/ha de calcário dolomítico, incorporado a aproximadamente $20 \mathrm{~cm}$ de profundidade, por meio de gradagem, ficando, a área, em repouso por 60 dias antes do plantio. A quantidade de calcário foi definida com base no critério de elevação da saturação por bases para $60 \%$, conforme recomendações da Comissão de Fertilidade do Solo do Estado de Minas Gerais (1989). Utilizou-se o delineamento experimental de blocos casualizados, com três repetições. Os tratamentos foram dispostos em esquema fatorial combinando-se três doses de $\mathrm{N}(0,8$ e $16 \mathrm{~g} /$ planta de $\mathrm{N})$; três doses de $\mathrm{P}(0,5 \mathrm{e}$ $10 \mathrm{~g} /$ planta de $\left.\mathrm{P}_{2} \mathrm{O}_{5}\right)$ e três doses de $\mathrm{K}(0,5 \mathrm{e}$ $10 \mathrm{~g} /$ planta de $\mathrm{K}_{2} \mathrm{O}$ ).

As mudas foram produzidas em casa de vegetação, obtidas de plantas selecionadas na Embrapa-CPAF-Acre, cuja biomassa seca destilada apresentou rendimento de óleo essencial de 5,2\%, com $92 \%$ de safrol no óleo essencial. O semeio foi realizado em $23 / 1 / 96$, para posterior repicagem em copos de plástico descartáveis de $180 \mathrm{~cm}^{3}$, quando as mudas apresentavam $1 \mathrm{~cm}$ de altura. $O$ substrato utilizado na sementeira foi a mistura de areia, esterco, e serragem de madeira semidecomposta, na proporção de 1:1:1. Nos copos de plástico foi acrescentado o terriço da mata, mantendo-se a proporção de 1:1:1:1 dos componentes do substrato

O plantio foi realizado entre os dias 10 e 13/7/96, no espaçamento de $0,60 \times 0,40 \mathrm{~m}$. Cada parcela foi constituída de 40 plantas, com dimensões de 3,0 × 3,2 m, sendo úteis as 18 plantas centrais. A adubação mineral foi realizada em covas, utilizando-se como fontes a uréia, superfosfato triplo e o cloreto de potássio. O P foi aplicado nas covas, em dose única; o $\mathrm{N}$ e o $\mathrm{K}$ foram aplicados parceladamente, em três vezes, aos 30, 45 e 60 dias após o transplante, em doses iguais. Foram realizadas capinas manuais e suplementação hídrica por meio de irrigação por aspersão. As quantidades de água aplicadas foram calculadas e adaptadas de acordo com os períodos de maior ocorrência de chuvas.

Foram efetuados dois cortes, aos 6 e 12 meses após o transplantio, a $40 \mathrm{~cm}$ de altura do nível do solo. Em seguida, a matéria fresca foi pesada e retirada uma amostra que foi acondicionada em saco de papel e colocada em estufa com ventilação forçada, a $60^{\circ} \mathrm{C}$, até atingir peso constante. A produção de óleo essencial foi determinada a partir de uma amostra de $25 \mathrm{~g}$ de folhas e ramos secados à sombra, até a umidade de $12 \%$. O processo utilizado foi a microdestilação de água e vapor com utilização de "Clevenger" e coobação de águas condensadas. 
Os caracteres avaliados foram as produções de matéria seca de ramos mais folhas (MSRF) e óleo essencial (OE) obtidas nos dois cortes realizados.

Os resultados foram submetidos a análise de variância, em que cada ensaio foi analisado individualmente Considerou-se inicialmente o modelo completo $\mathrm{Y}_{\mathrm{i}}=\beta_{\mathrm{o}}+\beta_{1} \mathrm{~N}_{\mathrm{i}}+\beta_{2} \mathrm{P}_{\mathrm{i}}+\beta_{3} \mathrm{~K}_{\mathrm{i}}+\beta_{4} \mathrm{~N}^{2}{ }_{\mathrm{i}}+\beta_{5} \mathrm{P}_{\mathrm{i}}+\beta_{6} \mathrm{~K}_{\mathrm{i}}{ }_{\mathrm{i}}+$ $\beta_{7} \mathrm{~N}_{\mathrm{i}} \mathrm{P}_{\mathrm{i}}+\beta_{8} \mathrm{~N}_{\mathrm{i}} \mathrm{K}_{\mathrm{i}}+\beta_{9} \mathrm{P}_{\mathrm{i}} \mathrm{K}_{\mathrm{i}}+\beta_{10} \mathrm{~N}_{\mathrm{i}} \mathrm{P}_{\mathrm{i}} \mathrm{K}_{\mathrm{i}}+\varepsilon_{\mathrm{i}}$ onde

Ni, Pi e Ki: níveis de adubação;

$\mathrm{Y}_{\mathrm{i}}$ : produções de matéria seca de ramos mais folhas, e de óleo essencial

$\varepsilon_{\mathrm{i}}$ : erro experimental.

A partir desse modelo foram analisados vários outros possíveis, em que a escolha do mais adequado baseou-se nos seguintes critérios: significância do efeito da regressão e não-significância dos desvios da regressão (falta de ajustamento) avaliadas pelo teste F; significância dos coeficientes das equações de regressão testados pelo teste t; e coeficiente de determinação $\left(\mathrm{R}^{2}\right)$ acima de $80 \%$.

\section{Resultados e Discussão}

No solo que recebeu calcário, houve significância dos efeitos do $\mathrm{P}(\mathrm{P}<0,01)$ e do $\mathrm{K}(\mathrm{P}<0,05)$, e não houve efeito do $\mathrm{N}$ na produção de matéria seca de ramos mais folhas (MSRF) (Figura 1). A produção de MSRF cresceu linearmente com as doses de $\mathrm{K}$, enquanto o $P$ promoveu efeito quadrático, e o máximo MSRF (7.402 kg/ha) obtido foi com a dose de

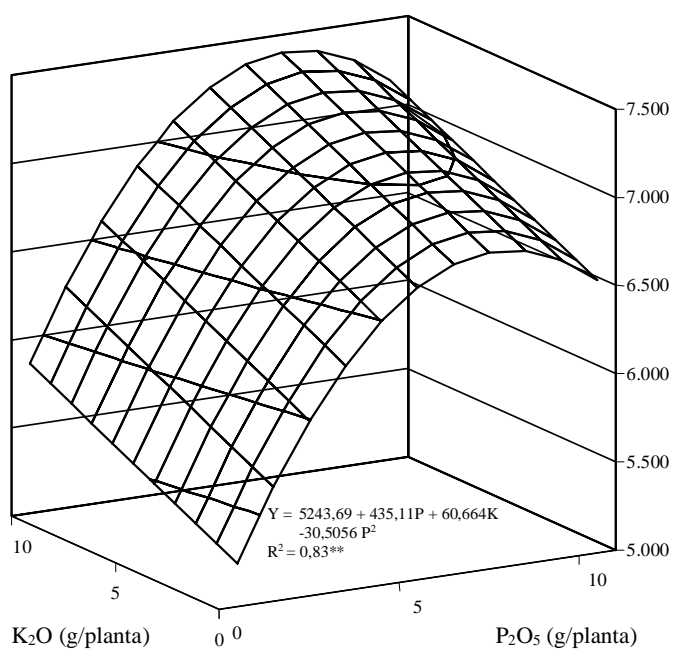

Figura 1. Produção de matéria seca de ramos mais folhas de pimenta-longa, em razão das doses de $\mathrm{P}$ e $\mathrm{K}$, em solo que recebeu calcário. Rio Branco, AC, 1997 $(* *$ : significativo a $1 \%)$
$7,1 \mathrm{~g} /$ planta de $\mathrm{P}_{2} \mathrm{O}_{5}$ quando se utilizou a maior dose de $\mathrm{K}\left(10 \mathrm{~g} /\right.$ planta $\left.\mathrm{K}_{2} \mathrm{O}\right)$. Tal produtividade, quando comparada com a obtida na ausência de adubação com P e K $(5.244 \mathrm{~kg} / \mathrm{ha})$, indica que a adubação com $\mathrm{P}$ e K proporcionou aumento de $41 \%$ na produtividade de biomassa seca.

No Estado do Pará, Brasil \& Viégas (1998) realizaram estudo semelhante em solo calcareado, utilizando as mesmas doses de N, P e K, porém em Latossolo Amarelo, textura média. As doses de $\mathrm{N}$ empregadas pelos autores promoveram aumento quadrático na produção de matéria seca da parte aérea apenas na interação com $5 \mathrm{~g} /$ planta de $\mathrm{K}_{2} \mathrm{O}$ e de $\mathrm{P}_{2} \mathrm{O}_{5}$, e aumento linear na interação com $5 \mathrm{e} 0 \mathrm{~g} /$ planta de $\mathrm{K}_{2} \mathrm{O}$ e de $\mathrm{P}_{2} \mathrm{O}_{5}$, respectivamente. Já as doses de $\mathrm{K}$ promoveram efeito quadrático apenas nas interações que utilizaram 16 e $0 \mathrm{~g} /$ planta de $\mathrm{N}$ e de $\mathrm{P}_{2} \mathrm{O}_{5}$, respectivamente, e 5 e 8 g/planta de $\mathrm{N}$ e de $\mathrm{P}_{2} \mathrm{O}_{5}$, respectivamente.

Esses resultados são discordantes dos obtidos neste trabalho, no qual não se verificou nenhuma interação significativa com o $\mathrm{N}$ na produção de MSRF, e as doses de $\mathrm{K}$ empregadas promoveram aumento linear. Provavelmente, como o solo utilizado neste trabalho (Argissolo Vermelho-Escuro textura argilosa) apresenta características física e química diferentes do solo usado por Brasil \& Viégas (1998), é de se esperar efeitos diferenciados da adubação com N, P e K sobre a produtividade da pimenta-longa.

Na produção de óleo essencial (OE), os efeitos do N, P e K não foram significativos. Apenas no desdobramento da interação N x P verificou-se que, na ausência de adubação com $\mathrm{P}$, as doses de $\mathrm{N}$ promoveram aumento linear na produção de $\mathrm{OE}$, enquanto na ausência de $\mathrm{N}$ as doses de $\mathrm{P}$ apresentaram efeito quadrático, e a máxima produtividade $(196 \mathrm{~L} / \mathrm{ha}$ de óleo essencial) foi alcançada com a dose de $5,78 \mathrm{~g} /$ planta $\mathrm{P}_{2} \mathrm{O}_{5}$ (Figura 2).

No experimento que não recebeu calcário, os efeitos do $\mathrm{N}$ e do $\mathrm{P}$ foram significativos a $1 \%$ de probabilidade na produção de MSRF e não-significativos os do K. A produção de MSRF foi independente das doses de K (Figura 3). As doses de N e P promoveram efeito quadrático na produção de MSRF, em que a resposta máxima $(5.920,4 \mathrm{~kg} / \mathrm{ha})$ seria obtida com a dose de 10,22 g/planta de $\mathrm{Ne}$ 9,62 g/planta de $\mathrm{P}_{2} \mathrm{O}_{5}$.

Na produção de óleo essencial, apenas os efeitos do $\mathrm{N}$ e do $\mathrm{P}$ apresentaram efeito quadrático e linear 

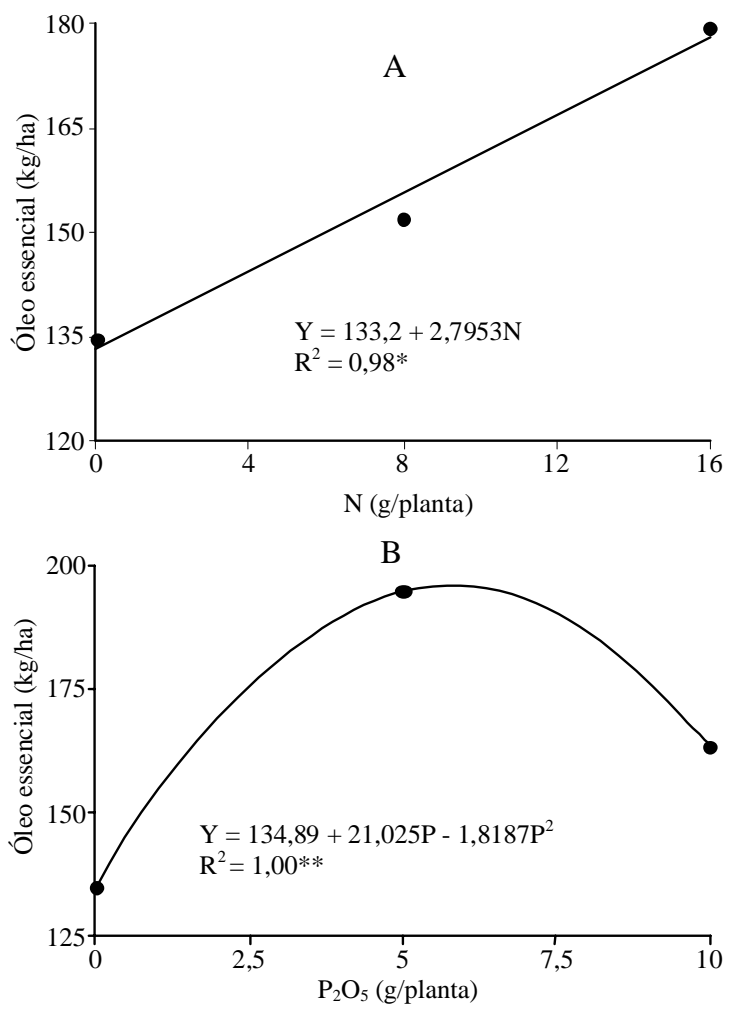

Figura 2. Produção de óleo essencial de pimenta-longa, em razão das doses de $\mathrm{N}$, na ausência de $\mathrm{P}(\mathrm{A})$; e das doses de $\mathrm{P}$, na ausência do $\mathrm{N}$ (B), em solo que recebeu calcário. Rio Branco, AC, 1997 (*,**: significativo a 5\% e $1 \%$, respectivamente)

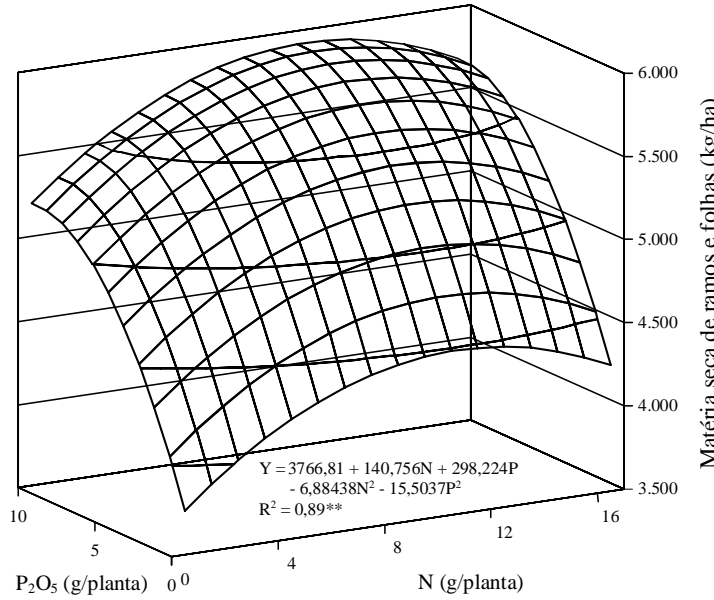

Figura 3. Produção de matéria seca de ramos mais folhas de pimenta-longa, em razão das doses de $\mathrm{N}$ e $\mathrm{P}$, em solo que não recebeu calcário. Rio Branco, AC, 1997 (**: significativo a $1 \%$ ). significativo a 5\% e 1\%, respectivamente, e não se verificou nenhuma interação significativa. A produção de $\mathrm{OE}$ aumentou linearmente com as doses de $\mathrm{P}$, o que indica que as doses usadas não foram suficientes para se obter a produção máxima (Figura 4). Já o aumento das doses de $\mathrm{N}$ apresentou efeito quadrático, em que a máxima produção seria obtida com a dose de 9,38 g/planta de N.

Em ambos os ensaios, com ou sem a aplicação de calcário, a adubação com $P$ foi a que apresentou efeito mais pronunciado no aumento da produção de MSRF, o que já era esperado por causa do baixo teor desse nutriente no solo utilizado nos ensaios $\left(1,0 \mathrm{mg} / \mathrm{dm}^{3}\right)$. Segundo Malavolta et al. (1989), é comum verificar, em solos com severa deficiência em um determinado nutriente, como neste caso o P, resposta significativa da planta quando de sua aplicação na adubação. O P é um dos fatores mais limitantes
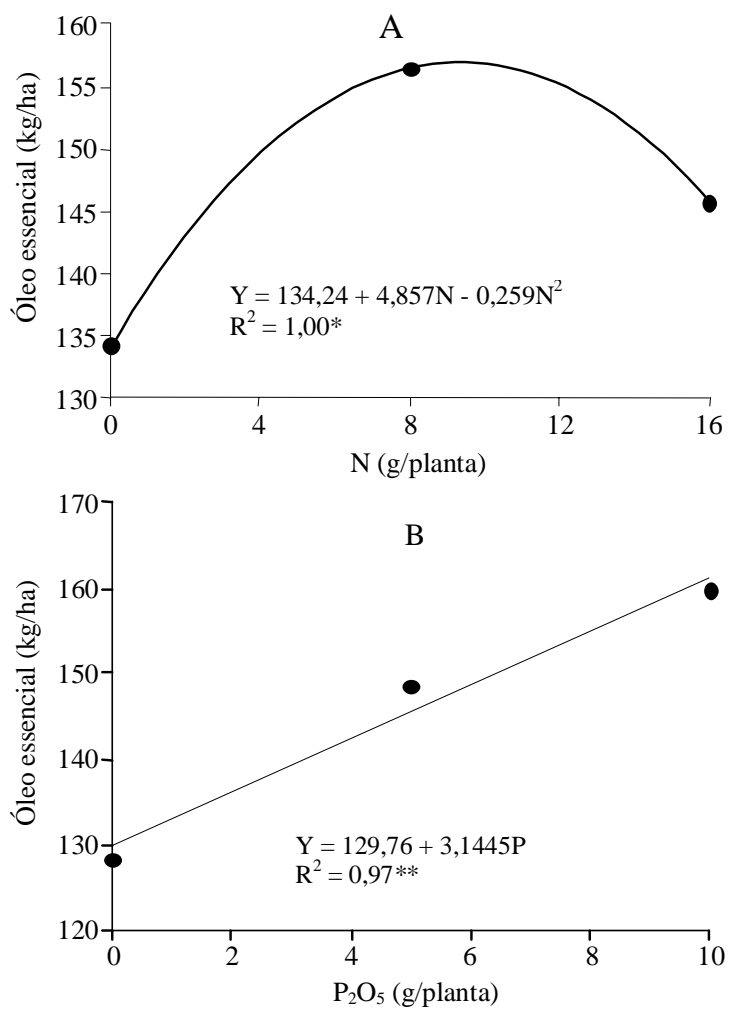

Figura 4. Produção de óleo essencial de pimenta-longa em razão das doses de $\mathrm{N}$ (A) e $\mathrm{P}(\mathrm{B})$, em solo que não recebeu calcário. Rio Branco, AC, 1997 $\left({ }^{*},{ }^{* *}\right.$ : significativo a $5 \%$ e $1 \%$, respectivamente $)$ 
para o desenvolvimento das culturas nos solos do Acre (Amaral \& Souza, 1998), e deve receber atenção especial no manejo da adubação da pimenta-longa, haja vista seu acentuado efeito na produção de biomassa seca.

De maneira geral, as médias obtidas nas produções de MSRF e OE foram maiores no ensaio cujo solo foi calcareado $(6.451 \mathrm{~kg} / \mathrm{ha}$ e $167 \mathrm{~L} / \mathrm{ha}$, respectivamente), do que as obtidas no solo não calcareado $(5.003 \mathrm{~kg} / \mathrm{ha}$ e $145 \mathrm{~L} / \mathrm{ha}$, respectivamente). Entretanto, as diferenças não foram de grande magnitude, principalmente em relação ao $\mathrm{OE}$. As áreas de ocorrência natural de pimenta-longa concentram-se no vale do rio Acre, sendo, em sua maioria, solos Podzólicos Vermelho-Amarelos e Podzólicos Amarelo distróficos, ácidos (Amaral \& Souza, 1998; Rocha Neto et al., 1999); dessa forma, é de se esperar que a espécie tenha certa tolerância quando cultivada em solos ácidos.

\section{Conclusões}

1. O P é o nutriente de efeito mais pronunciado no aumento da produção de matéria seca de ramos e folhas de pimenta-longa.

2. As doses de P promovem efeito quadrático sobre a produção de matéria seca de ramos e folhas, em solos que receberam ou não calcário.

3. Em solo não calcareado, as doses de N promovem efeito quadrático sobre as produções de matéria seca de ramos e folhas e de óleo essencial.

\section{Referências}

AMARAL, E. F. do; SOUZA, A. N. de. Avaliação da fertilidade do solo no sudeste acreano: o caso do $\mathrm{PED} / \mathrm{MMA}$ no Município de Senador Guiomard. Rio Branco Embrapa-CPAF-Acre, 1998. 32 p. (Embrapa-CPAF-Acre. Documentos, 26).

BRASIL, E. C.; VIÉGAS, I. de J. M. Efeito da adubação mineral na produção de matéria seca de pimenta-longa (Piper hispidinervum). Belém : Embrapa-CPATU, 1998 4 p. (Embrapa-CPATU. Pesquisa em Andamento, 180).

COMISSÃO DE FERTILIDADE DO SOLO DO ESTADO DE MINAS GERAIS (Lavras, MG). Recomendação para uso de corretivos e fertilizantes em Minas Gerais: 4⿳亠口冋 aproximação. Lavras, 1989. 176 p

COSTA, R. S. C. da; RODRIGUES, J. E. L. F.; JORGE, H. D.; BARBOSA, E. A. Adubação de pimenta-do-reino em Porto Velho-RO. Porto Velho : Embrapa-UEPAE de Porto Velho, 1989. 4 p. (Embrapa-UEPAE de Porto Velho. Pesquisa em Andamento, 116).

FAGERIA, N. K.; STONE, L. F.; SANTOS, A. B. dos Maximização da eficiência de produção das culturas. Brasília : Embrapa-CNPAF, 1999. 294 p.

MAIA, J. G.; SILVA, M. L. da; LUZ, A. I. R.; ZOGHBI, M. das G. B.; RAMOS, L. S. Espécies de Piper da Amazônia ricas em safrol. Química Nova, São Paulo, v. 10, n. 3, p. 200-204, 1987.

MALAVOLTA, E.; VITTI, G. C.; OLIVEIRA, S. A. Avaliação do estado nutricional das plantas: princípios e aplicações. Piracicaba : POTAFOS, 1989. 201 p.

PIMENTEL, F. A.; SOUSA, M. de M. M.; SÁ, C. P. de; CABRAL, W. G.; SILVA, M. R. da; PINHEIRO, P. S. N.; BASTOS, R. M. Recomendações básicas para o cultivo da pimenta-longa (Piper hispidinervum) no Estado do Acre. Rio Branco : Embrapa-CPAF-Acre, 1998. 9 p. (Embrapa-CPAF-Acre. Circular Técnica, 28).

RELATÓRIO TÉCNICOANUALDO CENTRODE PESQUISA AGROFLORESTAL DO ACRE. Rio Branco Embrapa-CPAF-Acre, 1992. 64 p. Edição Especial.

ROCHANETO, O. G. da; OLIVEIRA JÚNIOR, R. C. de; CARVALHO, J. E. U. de; LAMEIRA, O. A.; SOUSA, A. R. de; MARADIAGA, J. B. G. Principais produtos extrativos da Amazônia e seus coeficientes técnicos Brasília : IBAMA/Embrapa-CNPT, 1999. 78 p.

SILVA, M. H. L. da. Tecnologia de cultivo e produção racional de pimenta-longa, Piper hispidinervum C. DC. Rio de Janeiro : UFRRJ, 1993. 87 p. Dissertação de Mestrado.

VELOSO, C. A. C.; KATO, O. R.; ALBUQUERQUE, F. C. de; BRANDÃO, G. R. Adubação química de cultivares de pimenta-do-reino (Piper nigrum L.) em Latossolo Amarelo da rodovia Transamazônica. Belém : Embrapa-UEPAE de Belém, 1988. 6 p. (Embrapa-UEPAE de Belém. Comunicado Técnico, 1). 